\title{
Freeze-thaw Caenorhabditis elegans freeze- thaw stress response is regulated by the insulin/IGF-1 receptor daf-2
}

\author{
Jian-Ping $\mathrm{Hu}^{1+}$, Xiao-Ying $\mathrm{Xu}^{1+}$, Li-Ying Huang ${ }^{1}$, Li-shun Wang ${ }^{2^{*}}$ and Ning-Yuan Fang ${ }^{1 *}$
}

\begin{abstract}
Background: Adaption to cold temperatures, especially those below freezing, is essential for animal survival in cold environments. Freezing is also used for many medical, scientific, and industrial purposes. Natural freezing survival in animals has been extensively studied. However, the underlying mechanisms remain unclear. Previous studies demonstrated that animals survive in extremely cold weather by avoiding freezing or controlling the rate of ice-crystal formation in their bodies, which indicates that freezing survival is a passive thermodynamic process.
\end{abstract}

Results: Here, we showed that genetic programming actively promotes freezing survival in Caenorhabditis elegans. We found that daf-2, an insulin/IGF-1 receptor homologue, and loss-of-function enhanced survival during freeze-thaw stress, which required the transcription factor daf-16/FOXO and age-independent target genes. In particular, the freeze-thaw resistance of daf-2(rf) is highly allele-specific and has no correlation with lifespan, dauer formation, or hypoxia stress resistance.

Conclusions: Our results reveal a new function for daf-2 signaling, and, most importantly, demonstrate that genetic programming contributes to freezing survival.

Keywords: C. elegans, Freeze-thaw stress response, Insulin/IGF-1 receptor daf-2, Transcription factor daf-16/FOXO

\section{Background}

Cold temperature is a critical environment stimulus to animals. Subzero temperatures especially may adversely affect animals by direct lethal effects and damage caused by ice formation [1]. The ability of animals to sense and respond to cold temperatures, even below freezing, is essential for survival is cold environments. Freezing is also widely used for many medical, scientific, and industrial purposes, such as strain preservation and organ preservation. Understanding how to enhance survival and maintain normal physiological functions in the presence of freeze stress is critical for animals in nature and human research.

Natural freezing survival in animals has been extensively studied. Previous studies demonstrated that animals

\footnotetext{
*Correspondence: lishunwang@fudan.edu.cn; fangnysh@126.com

${ }^{\dagger}$ Equal contributors

${ }^{2}$ The Division of Translational Medicine, Minhang Hospital, Fudan University, Shanghai, China

${ }^{1}$ The Department of Geriatrics, Ren-Ji Hospital, Shanghai Jiao-Tong University School of Medicine, Shanghai, China
} C Biomed Central

(c) 2015 Hu et al. Open Access This article is distributed under the terms of the Creative Commons Attribution 4.0 International License (http://creativecommons.org/licenses/by/4.0/), which permits unrestricted use, distribution, and reproduction in any medium, provided you give appropriate credit to the original author(s) and the source, provide a link to the Creative Commons license, and indicate if changes were made. The Creative Commons Public Domain Dedication waiver (http://creativecommons.org/publicdomain/zero/1.0/) applies to the data made available in this article, unless otherwise stated. survive in extremely cold weather by avoiding freezing or [2], which indicates that freezing survival is a passive thermodynamic process. However, response to freezethaw stress and other biological phenomena, such as longevity and hypoxia resistance, may be genetically programmed. Because it is a powerful model in molecular genetics, the nematode Caenorhabditis elegans is suitable for studying genetic response to freeze-thaw stress.

Insulin/insulin-like growth factor 1 (IGF-1)-like signaling is the best-characterized pathway that regulates the lifespan and other stress-resistance traits of C. elegans. The insulin/IGF-1 receptor homologue daf-2 activates a conserved phosphatidylinositol-3-OH kinase $(\mathrm{PI}(3) \mathrm{K}) / 3$ phosphoinositide-dependent kinase-1 (PDK1)/Akt signal transduction pathway, which prevents FOXO transcription factor daf-16 entry into the nucleus [3-5]. daf-2 reduction/loss-of-function ( $\mathrm{rf}$ ) produces a longer lifespan [6]. Conversely, the PTEN phosphatase homologue, daf-18(rf), suppresses life-span extension induced by daf-2(rf) $[7,8]$. 
Insulin/IGF-1 signaling is also involved in formation of dauer larvae, which have an alternative, developmentally arrested third larval stage (L3) [9]. In unfavorable environments, such as crowding or food shortage, insulin/IGF-1 signaling or transforming growth factor-beta (TGF- $\beta$ ) is suppressed; unliganded nuclear receptor DAF-12 regulates dauer diapause [10]. Insulin/IGF-1 signaling is also involved in other stress tolerances, including oxidative stress, ultraviolet light, heat shock, or hypoxia stress. The longevity and stress tolerance produced by insulin/IGF-1 signaling mutants require nuclear translocation or nuclear activity of daf-16 [11]. Savory et al. [12] showed that daf16 is important for delta-9 desaturase gene expression, which is important for survival at low temperatures. Ohta [13] showed that the insulin-signaling pathway in the intestines and neurons is essential for temperature experience-dependent cold tolerance in animals. Animal survival in freezing conditions is a more complicated phenomenon than in cold temperatures without ice formation. Organs can be injured during freezing by physical factors, such as ice-crystal formation, dehydration, and cold [14]. Moreover, animals can also suffer biochemical damage, such as oxidative stress or hypoxia stress. [15]

To date, few studies have addressed the genes that regulate freezing tolerance or survival. We investigated the roles of $d a f-2(\mathrm{rf})$ in freeze-thaw stress-response regulation. We exposed daf-2(e1370) and other rf strains to freeze-thaw stress to identify daf-2(rf)-improved freeze-induced mortality and cell damage. Then, we tested other daf-2(rf) alleles with various phenotypic severities and performed molecular analysis to determine which signaling is involved. We performed the experiments through both genetic and morphological analyses with different $C$. elegans mutants.

\section{Results}

Insulin/IGF-1 receptor homologue daf-2(rf) regulates freeze-thaw stress survival

To investigate the role of $d a f-2(\mathrm{rf})$ in freezing tolerance, we evaluated the survival rate of daf-2(e1370) and wildtype (N2) strains exposed to freeze-thaw stress. Two-dayold adults were exposed to $-80{ }^{\circ} \mathrm{C}$ for $8 \mathrm{~min}$ and then thawed in a water bath at $30{ }^{\circ} \mathrm{C}$; results showed that the daf-2(e1370) strain had a significantly increased survival rate compared with the $N 2$ strain $(\mathrm{p}<0.01$; Fig. 1a). To confirm the results of enhanced freezing survival produced by the reduction of $d a f-2 /$ insulin-like signaling, we evaluated the survival rate of $N 2$ animals with daf-2 RNAi interference or $\mathrm{IGF}_{1} \mathrm{R}$ inhibitor treatment. Animals with both daf-2 RNAi inactivation and $\mathrm{IGF}_{1} \mathrm{R}$ inhibitor treatment had enhanced survival rates after freeze-thaw treatment ( $<<0.01$; Fig. 1a).

At high freezing rates, intracellular freezing occurs, which can lead to cell damage, mainly by ice-crystal formation $[15,16]$. To reduce ice-crystal formation and cell damage, cells and organisms can be cryopreserved by slowly lowering the temperature until deep freezing temperature. daf-2(e1370) and $N 2$ animals were gradually cooled at a rate of about $-1{ }^{\circ} \mathrm{C} / \mathrm{min}$ (Additional file 1 : Figure S1). Moreover, we also found that daf-2(e1370) animals had higher survival than N2 at any growth stage. This result indicates that survival of daf-2(rf) animals following freeze-thaw damage is not stage- or age -specific (Fig. 1, Additional file 1: Figure S1A-C). We also found that the survival rate of L3/L4-stage worms was dramatically decreased compared with L1/ L2-stage worms (Fig. 1b, Additional file 1: Figure S1A-B). This result indicates that freezing tolerance changed with development and age.

\section{Freezing-induced behavioral defects and cell defects blocked by daf-2(rf)}

Maintaining physiological functions in freezing temperatures is challenging; therefore, evaluation of response to different freezing conditions is important. Under freeze-thaw conditions, animals frozen at $0{ }^{\circ} \mathrm{C}$ and thawed in $30{ }^{\circ} \mathrm{C}$ water baths did not differ in survival rate (Additional file 2: Figure S2.), which indicates that the animals' physiological function loss is mainly caused by freezing rather than thawing. daf-2(e1370) animals survived and fully recovered locomotion after freeze-thaw stress. N2 animals displayed significant locomotion defects after recovery from some stress (Fig. 2).

To investigate cell defects from freezing and protection by daf-2(rf), we examined the muscle cell morphology of animals exposed to freeze-thaw stress. Freezing caused striking nuclear fragmentation in muscle myocytes. RNAi inactivation of $d a f-2$ and downstream genes age, $p d k-1$ maintained intact nuclei and protected myocytes from both nuclear fragmentation and death [Fig. 3].

daf-2 allelic specification for freeze-thaw stress survival is not a consequence of lifespan, dauer formation, or other stress resistance mechanisms

daf-2(rf) alleles tend to promote a prolonged lifespan and form dauer larvae. Consequently, daf-2(rf) mutants are always resistant to harsh environments [17]. To analyze whether freeze-thaw survival is correlated with lifespan, we tested freezing survival rates associated with 11 daf-2 alleles' (Fig. 4). The freeze-thaw stress survival phenotypes were not well correlated with lifespan $(\mathrm{r}=0.538 ; \mathrm{p}=0.088)$.

We found that daf-2(e1370) worms, which did not have the longest lifespan, had the highest freeze-thaw stress survival, followed by e1391, e1371, e979, and $m 579$. Five alleles that were weak or produced no increased freezing survival had significantly increased lifespans as long as or longer than e1370 [17]. Similarly, 


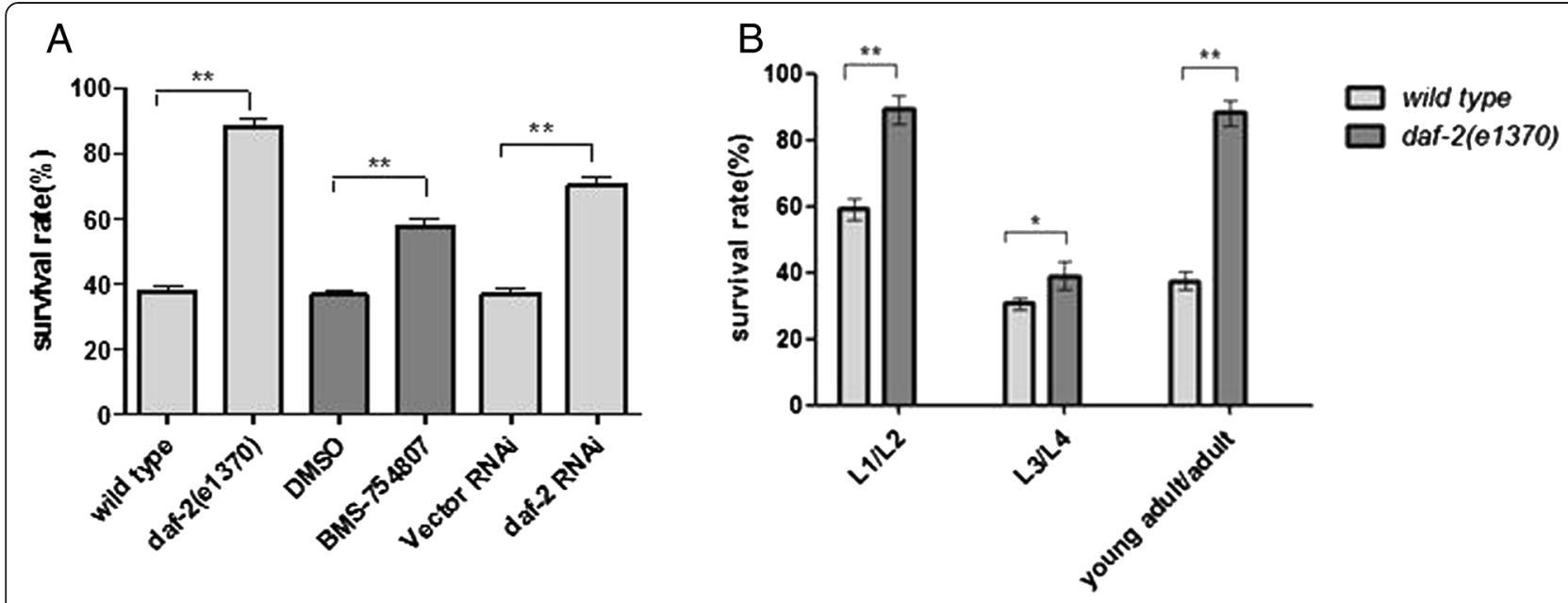

Fig. 1 Enhanced freeze-thaw stress survival induced by IGF-1 receptor/daf-2 reduction-of-function. a daf-2(e1370rf), IGF-1 receptor inhibitor (BMS-754807) treatment, and daf-2 RNAi increased freeze-thaw stress survival compared with corresponding wild-type control animals. Every assay was repeated five times ( $n>170-203)$ in the same condition. Statistical significance was assessed by the Mann-Whitney test. ${ }^{* *} p<0.01$. $\mathbf{b}$ daf-2(e1370) enhanced freeze-thaw stress survival at different growth stages, from L1 to adult stages. Survival rates were assayed $6 \mathrm{~h}$ after recovering from freeze-thaw stress (L1/L2- and L3/L4-stage animals were exposed to freezing for $16 \mathrm{~min}$, and young adult/adult animals were exposed to freezing for 8 min at - $80{ }^{\circ} \mathrm{C}$ ). Every assay was repeated five times ( $n>180-286)$. Statistical significance was assessed by the Mann-Whitney test. ${ }^{*} p<0.05,{ }^{* *} p<0.01$

one allele that did not increase survival $(\mathrm{m} 41)$ and two weaker alleles (e1391 and e979) produced stronger Daf-c phenotypes than $e 1370$, and one allele that did not increase survival $(e 1368)$ produced the same Daf-c phenotype as $e 1370[18,19]$. With regard to stress resistance, four daf-2 alleles (e1391,e979, e579, and $m 596)$ were

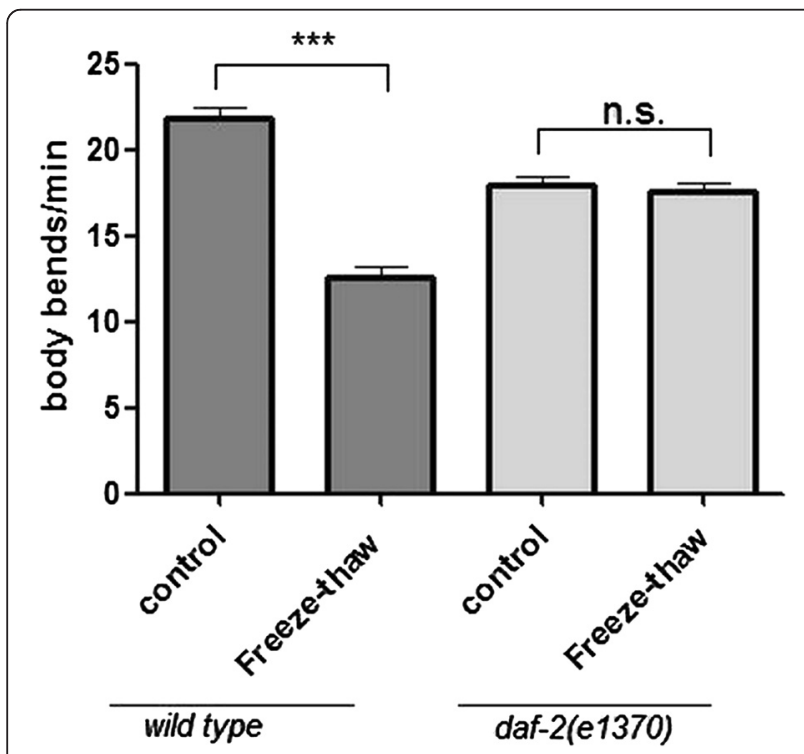

Fig. 2 Behavioral effects of freeze-thaw stress in wild-type (N2) and daf-2(e1370rf) strains. Locomotion rate was quantified as the number of body bends per min after recovery from freeze-thaw stress. Locomotion of N2 $(n=10)$ was significantly reduced after recovery from freeze-thaw stress; daf-2(e1370) remained unchanged $(n=10)$. Statistical significance was assessed by the Mann-Whitney test. ${ }^{* * *} p<0.001$, n.s. - not significant significantly resistant to thermal stress [18] but had weaker or no increased freeze-thaw survival. daf-2 allele $e 579$ was significantly resistant to hypoxia stress but had weaker freeze-thaw survival. Three daf-2 alleles (e1371, e1391, and $e 979$ ) were weak or non-resistant to hypoxia stress but had higher freeze-thaw survival. daf-2(rf) freeze-thaw stress survival was highly allele-specific and did not appear to be a consequence of mechanisms that regulate lifespan, dauer formation, or other stress-resistance traits (Additional file 3: Figure S3).

The insulin-signaling pathway, but not dauer signaling, longevity genes, or trpa-1, is essential for freeze-thaw stress survival

To determine the molecules downstream of the insulin receptor involved freeze-thaw stress survival, we tested various mutants defective in the known insulin-signaling pathway (Fig. 5). Phenotypic analysis showed that mutants defective in the $d a f-2 /$ insulin receptor or its downstream molecules had abnormal enhancement or reduction of freeze-thaw stress survival (Fig. 5). Abnormal increments of freeze-thaw stress survival in $d a f-2$ and downstream molecules mutants were suppressed by mutation or RNAi in the daf-16/FOXO-type transcriptional factor (Fig. 6).

Because the insulin-signaling pathway and other signaling molecules are essential for dauer larva formation, we tested other molecular components, including TGF- $\beta$ and steroid hormonal signaling (Fig. 5) $[19,20]$. However, we did not observe a considerable increase in these mutants (Fig. 5). The results indicate that the daf-2/ insulin-signaling pathway, but not other dauer larva formation signaling, is essential for freezing survival. 


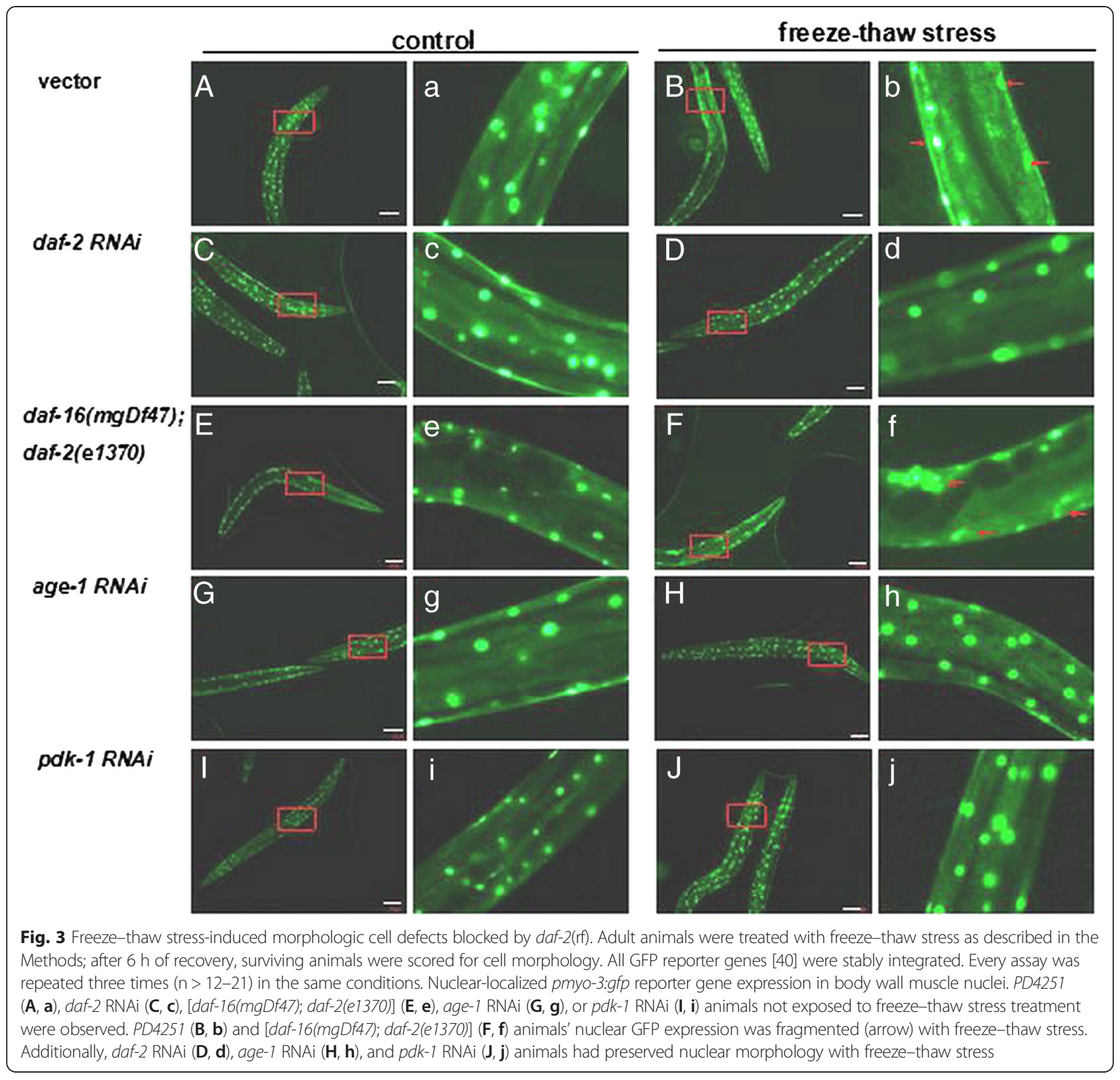

$d a f-2$ is the best-characterized longevity gene in $C$. elegans. We tested other longevity genes mutants, including eat-2, clk-1, and isp-1 mutants, and found that these animals did not have enhanced freezing survival compared with the N2 strain. Recently, it was reported that the cold receptor transient receptor potential (TRP) channel, which is encoded by trpa-1, plays a central role in ageing and stress response to cold temperatures [21]. However, no reduced freezethaw survival was observed in trpa-1 loss-of-function mutants. These results indicate that longevity genes and trpa-1 are not essential for freeze-thaw stress survival. daf-16 nuclear tranlsocation is not responsible for daf-2(rf)-enhanced freeze-thaw survival

To elucidate how daf-16 affects daf-2(rf) enhanced freeze-thaw stress survival, cellular distributions of a daf-16::GFP fusion protein were first categorized on a scale of 1 (unlocalized) to 3 (fully nuclear localized) (Fig. 7). Distributions were scored in daf-2 RNAi animals and compared with controls maintained at $20{ }^{\circ} \mathrm{C}$. As a positive control, daf-2 inactivation resulted in a marked translocation of $d a f-16$ to the nucleus (Fig. $7 \mathrm{a}-\mathrm{d}$, left histogram). With freeze-thaw stress, daf-16 nuclear translocation significantly increased in both daf-2 RNAi and control animals (Fig. 7e-h). However, the difference 


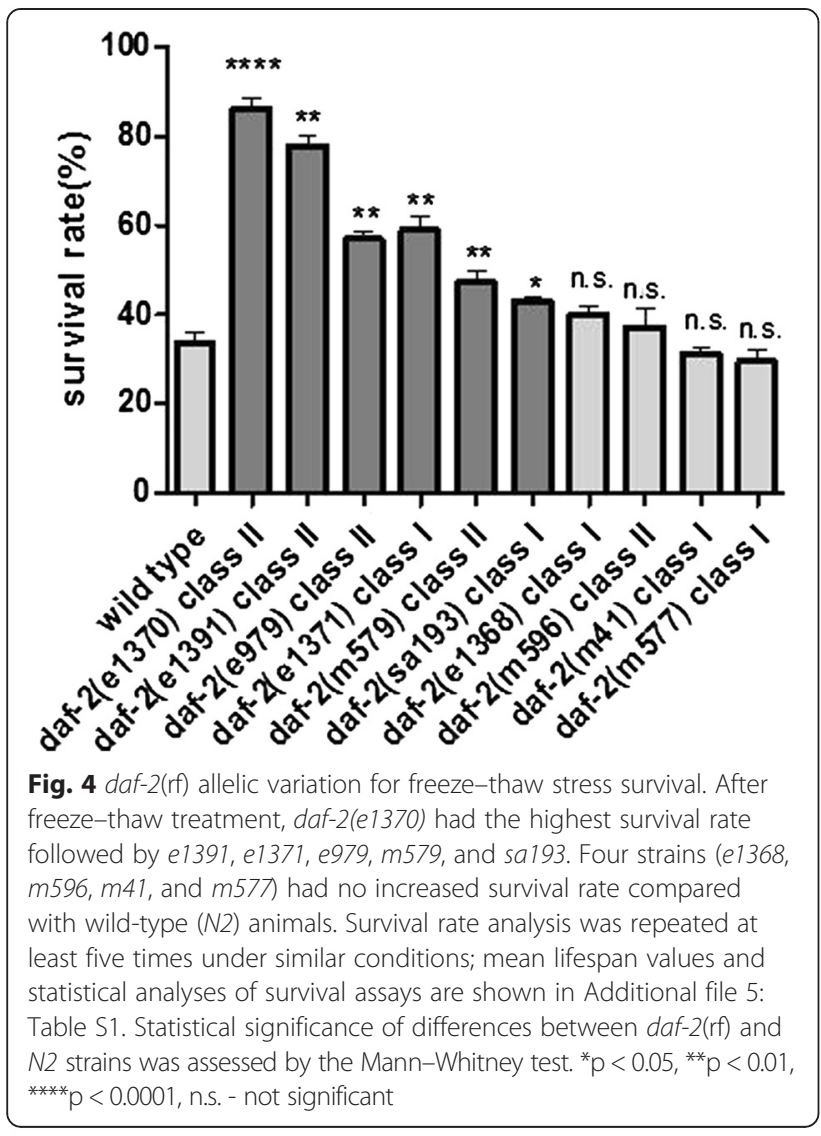

in daf-16 nuclear translocation between daf-2(rf) and control animals disappeared, because there was no significant discrepancy in the subcellular localization of daf-16::GFP (Fig. 7, right histogram). These observations indicate that altering the subcellular localization of daf-16 does not explain why daf-2(rf) has higher freezethaw stress survival than wild-type animals. We found that other mechanisms responsible for daf-2(rf) increased freezing survival.

To gather additional evidence to elucidate the nuclear activity of daf-16 responsible for daf-2(rf) increased freeze-thaw stress survival, we assayed daf-16 target genes. If the insulin pathway promotes daf-16 nuclear activity, this pathway should regulate the expression level of daf-16 target genes. We first examined the expression level of 21 direct transcriptional target genes of daf-16 that are also involved in ageing, larval arrest, or fat formation. We found that mRNA levels of C36A4.9 (acs-19, acetyl-CoA synthetase) and C46A10.7 (srh-99, class $\mathrm{H}$ chemoreceptor/olfactory receptor) were markedly upregulated in daf-2(e1370) worms (Additional file 4: Figure 4). Mice deficient in the homologous AceCS2 (acetyl-CoA synthetase 2) gene cannot maintain normal body temperatures when starved or fed a LC/HF diet [22]. In Drosophila, central and peripheral elements of the

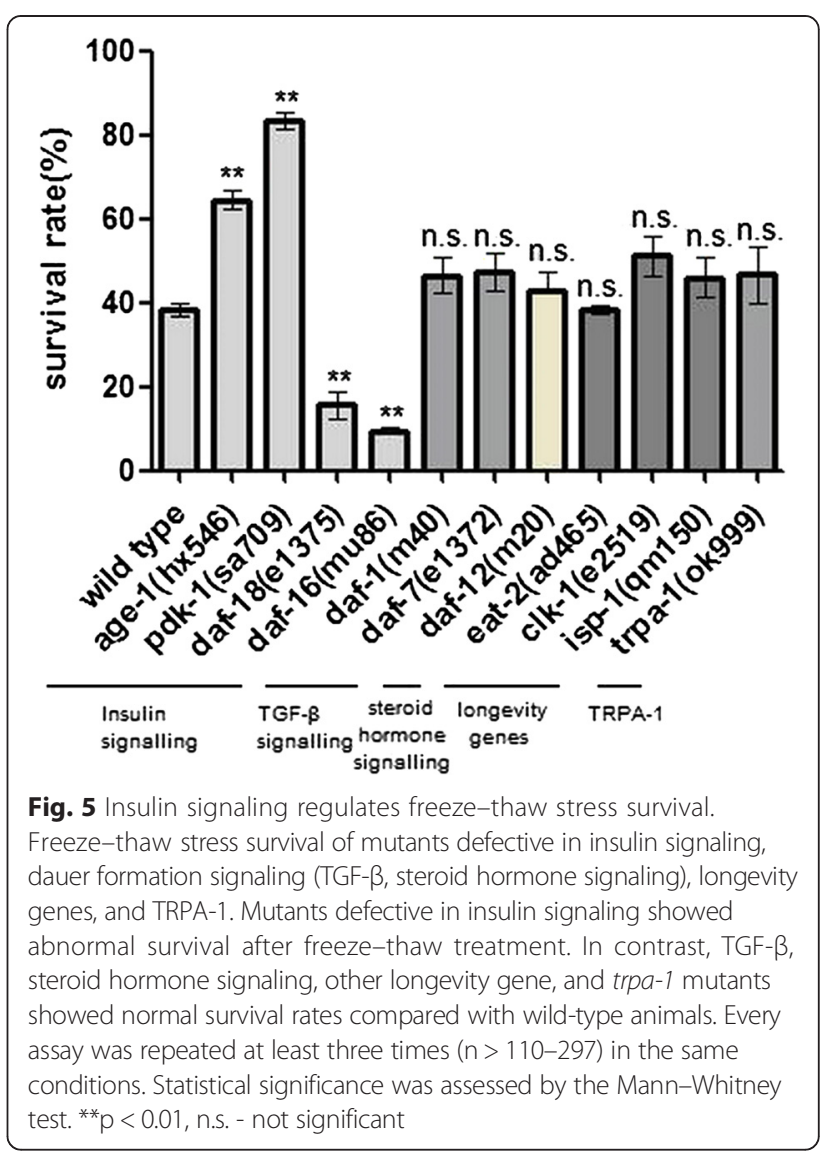

olfactory receptor system are responsible for temperature adaptation [23].

We infer that acs-19 or srh-99 may be required for freeze-thaw adaptation in C. elegans. For further elucidation, we assayed freeze-thaw survival rates with acs-19 and srh-99 RNAi in daf-2(e1370) and wild-type animals. We found that acs-19 and srh-99 RNAi significantly reduced daf-2(e1370) freeze-thaw survival, although there was still higher survival than in the daf-16;daf-2 double mutant (Fig. 8). This result indicates that the daf-16 target genes acs-19 and srh-99 are involved in the observed daf-2(rf) increased freeze-thaw stress survival. While, there is no evidence indicating other daf- 16 target genes sup-37 or lig-1 involved in daf-2(rf) increased freezing survival (Fig. 8).

\section{Discussion}

In this study, we revealed genetic regulation of freezethaw stress responses in C. elegans. Phenotypic analysis of genetic deletion strains revealed that insulin/IGF-1 receptor daf-2 controls both survival and behavior during freeze-thaw stress. daf-2 reduction improved freeze-thaw stress survival, locomotion, and muscle cell protection. $d a f-2$ (rf) freeze-thaw response is highly allele-specific and not a consequence of lifespan, dauer formation, or other 


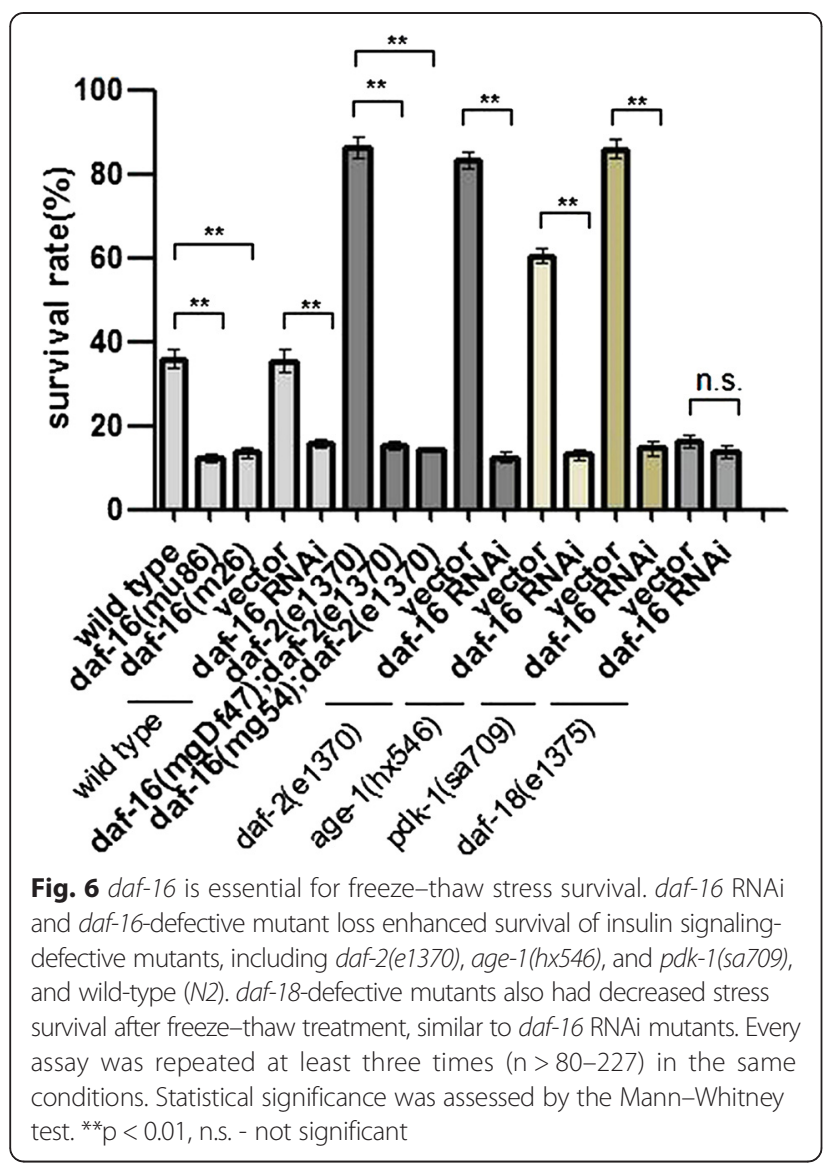

stress-resistance trait regulation. We also revealed that insulin signaling, but not TGF- $\beta$, are related to freeze-thaw survival. Steroid hormone signaling participated in daf2(rf) enhanced freeze-thaw stress regulation. daf-16::GFP cellular localization analysis and daf-16 target gene screening revealed that daf-16 regulated the target genes acs-19 and srh-99 but not daf-16 nuclear translocation or daf-2(rf) enhanced freeze-thaw stress survival.

In the past few decades, there has been rapid progress in our understanding of how physiological mechanisms can protect freeze injury [24]. By contrast, very little is known about how genetic regulation promotes freeze resistance. At least in C. elegans, freeze resistance is not purely a passive thermodynamic process. In this study, we characterized insulin/IGF-1 receptor daf-2, which regulates freeze-thaw stress survival improvement in $C$. elegans. Our results indicate that genetic programming actively contribute to enhanced survival and physiological function recovery from freezing conditions.

Previous work revealed that insulin signaling is required for lifespan regulation, dauer formation, and stress tolerance. Recent studies demonstrated that the insulinsignaling pathway or daf-16 is required for temperature experience-dependent cold tolerance of animals [12, 13]. To ascertain whether insulin signaling plays roles in regulating freeze resistance in very stressful conditions, we performed survival experiments with freeze-thaw stress. In these experiments, we confirmed that insulin signaling via the daf-16/FOXO pathway is essential for freeze-thaw survival. Reduction-of-function of insulin/IGF-R daf-2 protected muscle cell damage and promoted physiological activity recovery from freeze-thaw stress.

We found that freeze-thaw survival was daf-2(rf) allele-specific and is not a consequence of ageing, larval arrest, or other stress-resistance traits. Insulin signaling is also involved in dauer formation. To determine whether other dauer signaling participated in the freezethaw stress resistance, we assayed freeze-thaw survival of mutants. We found that dauer formation pathways, including TGF- $\beta$ and steroid receptor signaling, were not essential for freeze-thaw stress survival. In addition, daf-2 is the best-characterized longevity-regulated gene. Therefore, we also tested other freeze survival longevity gene mutants, including eat-2 [25], clk-1 [26], and isp-1 [27]. We found that these other longevity genes were not required for freeze-stress resistance. Low temperatures led to increased longevity, in which the cold receptor TRP channel encoded by trpa-1 is essential [21, 28]. Therefore, we tested the function of trpa-1 and found that loss-of-function mutant trpa-1 produced a normal phenotype compared with the wild-type strain. These results indicate that freeze-thaw survival is independent on cold receptor TRPA-1 signaling. These results are consistent with the daf-2(rf) allele relationship analysis between freeze-thaw survival and lifespan, larval arrest, and other stress-resistance traits. Freezing resistance may have an independent biological mechanism that differs from mechanisms that control ageing, dauer formation, and response to other stresses.

Finally, our results indicate that the daf-16 target genes C36A4.9 (acs-19) and C46A10.7 (srh-99) are required for freezing survival of daf-2(e1370). Previous studies demonstrated that acs-19 maintained core body temperatures of mice in fasting conditions [22], and olfactory receptor be acclimated to the environmental temperature of D. melanogaster [23]. Inactivation by RNAi of acs-19 or srh-99 contributed to both decreased fat storage and enhanced dauer formation of C. elegans [29]. It is possible that an unrecognized underlying mechanism contributes to both fat storage and dauer formation phenomena, which are related to freezing tolerance and survival. Nevertheless, our analyses did not exclude the contribution of additional mechanisms to freezing survival. acs-19 and srh-99 RNAi reduced freezing survival of daf-2(e1370); however, daf-2(e1370) acs-19 RNAi and daf-2(e1370) srh-99 RNAi mutants exhibited significantly higher survival compared with the daf-16 mutant, which indicates that additional daf-16 target genes or mechanisms must be involved. For example, 


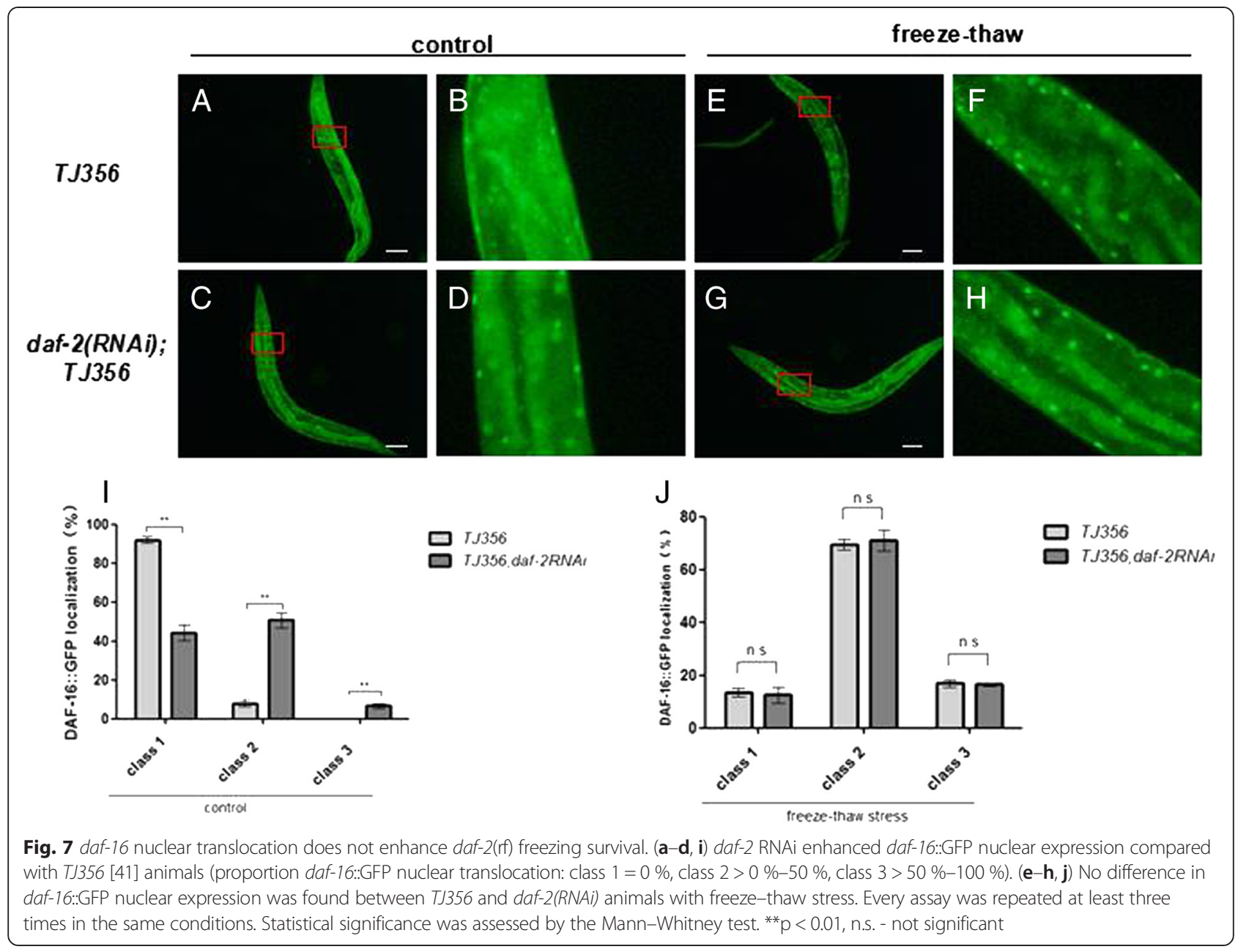

genes involved in the synthesis of trehalose [30-34], glycerol [30], heat-shock proteins [32, 35, 36], antioxidant enzymes [37], and $\Delta 9$ desaturase enzymes [12] could participate in freeze-thaw stress survival by enhancing cold tolerance of C. elegans and other species. Future studies are needed to identify other unknown genetic programming and additional components of the daf-2(rf)/ daf-16 pathway that ultimately may lead to a thorough understanding of how daf-2(rf) promotes freezing survival in C. elegans.

\section{Conclusion}

In conclusion, our mutant survival assay revealed that insulin/IGF-1 receptor daf-2 played important roles in freeze-thaw stress responses in C. elegans. Freezing resistance is $d a f-2$ allele-specific and not a consequence of ageing, dauer formation, and other stress-regulation traits. Reduction-of-function of daf-2 enhanced freezethaw survival, because it is dependent on insulin signaling pathway. The daf-16/FOXO-regulating target genes acs19 are srh-99 essential for daf-2(rf) enhanced freezing resistance. Considering that the insulin/IGF-1 receptor showed striking conservation across phylogeny [38, 39], our work indicates that a similar phenomenon may also occur in other organisms.

\section{Methods}

\section{Strain selection and maintenance}

Caenorhabditis elegans were maintained on nematode growth medium (NGM) agar plates seeded with OP50, which is a slow-growing Escherichia coli mutant. The strains used included N2, eat-2(ad465), isp-1(qm150), clk-1(qm30), daf-1(m40), daf-7(e1372), daf-12(rh286), daf-2(e1370), daf-2(e979), daf-2(e1391), daf-2(e1368), daf-2( e1371), daf-2(m596), daf-2(m577), daf2(m579), daf-2(Sa193), daf-2(m41), [daf-16(mgDf47); daf2(e1370)], TJ356(daf-16::GFP), and PD4251(pmyo-3::GFP). All strains were provided by the Caenorhabditis Genetics Center funded by the National Institutes of Health National Center for Research Resources. Unless otherwise stated, all strains were cultured at $15{ }^{\circ} \mathrm{C}$ to the $\mathrm{L} 4$ stage and then transferred to animals at $20^{\circ} \mathrm{C}$ for $2 \mathrm{~d}$. 


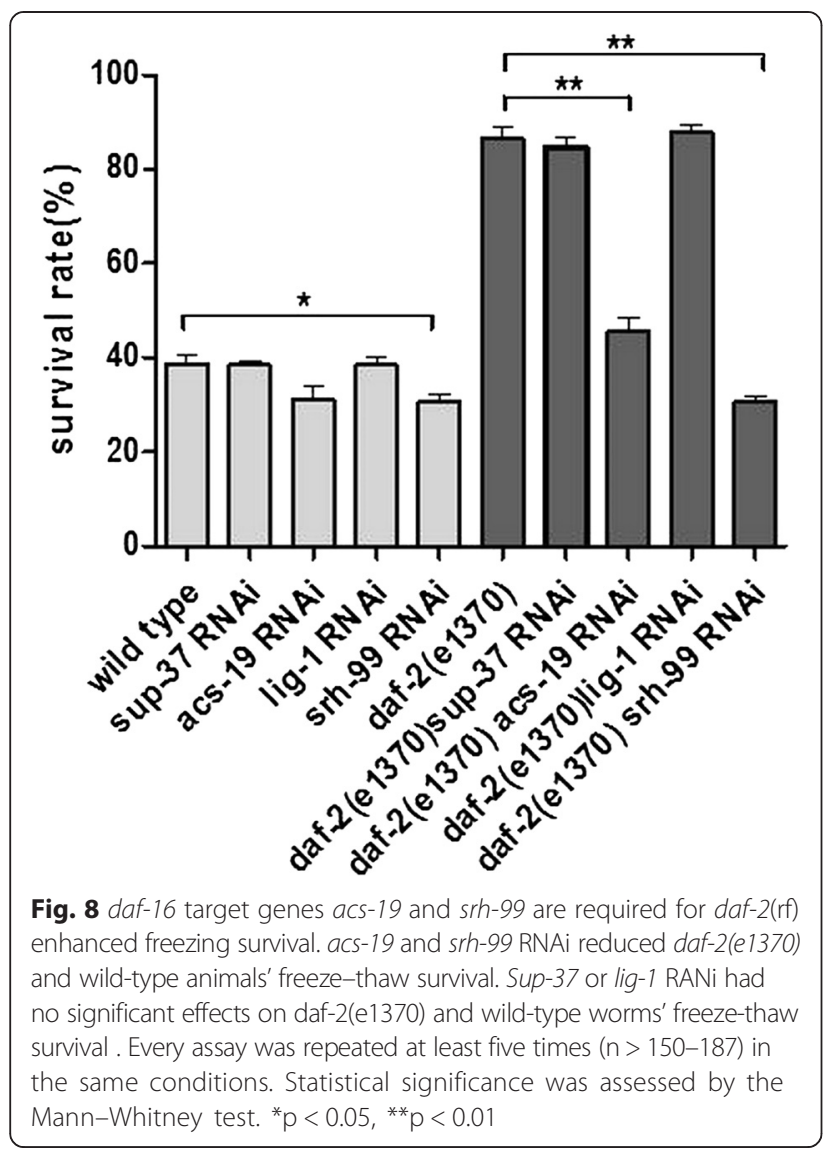

Freeze-thaw stress conditions

Adult animals (2 d after L4) on NGM plates were washed with M9 buffer and transferred into FACS tube with $1 \mathrm{ml}$ M9 buffer. Caenorhabditis elegans does not survive freezing very well, so to determine optimal freezing length for analyses, we placed animals from room temperature into $-80{ }^{\circ} \mathrm{C}$ for different amounts of times (4 min, $5 \mathrm{~min}, 6 \mathrm{~min}, 8 \mathrm{~min}, 12 \mathrm{~min}$, or $16 \mathrm{~min}$ ). At 4 min, there was no survival difference between $N 2$ and daf-2(e1370). Ice began to form at $5 \mathrm{~min}$, and all water froze in the tube at 6-7 $\mathrm{min}$; however, all animals, including $N 2$ and daf-2(rf), died at $16 \mathrm{~min}$. At $8 \mathrm{~min}$, there were significant freezing survival differences among mutants. Therefore, animals were treated with freezing stress at $-80{ }^{\circ} \mathrm{C}$ for $8 \mathrm{~min}$ for subsequent analyses. This experiment revealed that freezing stress damaged animals; it is possible that in the body, some cells did not completely freeze, but the freezing stress still injured animals.

Animals were exposed to freezing stress and then thawed at different temperatures $\left(0{ }^{\circ} \mathrm{C}\right.$ and $\left.30{ }^{\circ} \mathrm{C}\right)$ for different lengths of time $\left(10 \mathrm{~min}\right.$ for $0{ }^{\circ} \mathrm{C}$ and approximately $1 \mathrm{~min}$ for $30{ }^{\circ} \mathrm{C}$ ). We removed the tubes at the end of thawing process (when the ice was completely thawed), when the temperature in the tube is still $0{ }^{\circ} \mathrm{C}$.
All treated animals were placed on dry NGM plates for $6 \mathrm{~h}$ before determining mortality score with an optical microscope. L1-adult animals were used for the $d a f-2(\mathrm{rf})$ survival test for different stages.

\section{Programmed freezing conditions}

L1-adult animals on NGM plates were gently washed with M9 buffer. For the freezing procedure, a Cryo $1{ }^{\circ} \mathrm{C}$ freezing container was used with a gradient cooling rate of $-1{ }^{\circ} \mathrm{C} / \mathrm{min}$. Animals were transferred into freezing tubes with $0.9 \mathrm{ml}$ buffer and $0.9 \mathrm{ml} 30 \%$ glycerin. At different time points (every 20 or $40 \mathrm{~min}$ ), animals were thawed in a $30{ }^{\circ} \mathrm{C}$ water bath, and the survival rate was then assayed.

\section{Feeding RNAi}

dsRNA-expressing E. coli were streaked onto LB agar plates that contained ampicillin $\left(50 \mu \mathrm{g} \mathrm{ml}^{-1}\right)$ and tetracycline $\left(12.5 \mu \mathrm{g} \mathrm{ml}^{-1}\right)$, and then incubated at $37^{\circ} \mathrm{C}$ overnight. Bacteria were inoculated in $3 \mathrm{ml}$ LB liquid medium that contained only ampicillin $\left(100 \mu \mathrm{g} \mathrm{ml}{ }^{-1}\right)$ and then incubated at $37{ }^{\circ} \mathrm{C}$ overnight. All $3 \mathrm{ml}$ of culture was spun down, the supernatant poured off until $150 \mu \mathrm{l}$ was left $(20 \times$ concentrated culture), and pellets were resuspended. Then, $50 \mu \mathrm{l}$ of cells were resuspended to the center of RNAi plate (NGM/IPTG/ampicillin), allowed to dry (wrapped in aluminum foil), and induced overnight at room temperature (RNAi-seeded plates can be stored at room temperature for 2-3 d before use). Synchronized L1 worms were placed on each plate and incubated at 15 or $20^{\circ} \mathrm{C}$ until they reached the desired stage for further experiments.

\section{Scoring mortality}

Worms were scored on NGM plates after $6 \mathrm{~h}$ of recovery from the thawing procedure. Worms were prodded with a pick at least three times over approximately $10 \mathrm{~s}$; any that failed to move were counted as dead and removed from the plate.

\section{Morphologic cell defects}

The PD4251 reporter gene pmyo-3::gfp, which is located in body wall muscle nuclei, was assayed with a fluorescence microscope. pmyo-3::gfp expression of strain PD4251 by RNAi treatment with freezing and thawing was assayed.

Quantitation of locomotion rate by counting body bends

Ten animals were selected from fresh plates with fairly thin lawns, and one worm each was placed new plates. Assays started $24 \mathrm{~h}$ later $( \pm 1 \mathrm{~h})$. A 3-min timer was used to count number of body bends. Every time the part of the worm just behind the pharynx reached a maximum bend in the opposite direction from the bend last counted was considered one body bend. 


\section{daf-16 nucleus translocation}

TJ356 nuclear protein daf-16::GFP expression was assayed with a fluorescence microscope. daf-16::GFP expression of [daf-2(RNAi); TJ356] treated with freezing and thawing was assayed compared with that of TJ356.

\section{RNA isolation for qRT-PCR}

Total mRNA was extracted by TRIzol reagent (Invitrogen, Carlsbad, CA) from certain C. elegans and treated with RNase-free DNase (Promega, Madison, WI). Then, reverse transcription (RT) was performed with a TaKaRa RNA PCR kit (Takara, Dalian, China) following the manufacturer's instructions. The primers used are described in Additional file 5: Table S1 and sequenced in the DNA Sequencing Department of Biosune Systems Biology (Shanghai, China).

\section{mRNA reverse transcription and qRT-PCR}

One nanogram in vitro transcribed RNA was added to the RNA sample (500-1000 ng). DEPC $\mathrm{H}_{2} \mathrm{O}$ was added to the RNA sample to $29.5 \mu \mathrm{l}$. Then, $0.5 \mu \mathrm{l}$ each of $1 \mu \mathrm{g} / \mu \mathrm{l}$ random hexamer and $1 \mu \mathrm{g} / \mu \mathrm{l}$ poly dT were added. The mixture was incubated at $65{ }^{\circ} \mathrm{C}$ for $10 \mathrm{~min}$, immediately put on ice for $5 \mathrm{~min}$, and let stand at room temperature for $10 \mathrm{~min}$. Then, $18.5 \mu \mathrm{l}$ pre-mixture, which contained $2.5 \mu \mathrm{l} 10 \mathrm{mM}$ dNTP mix, $10 \mu \mathrm{l} 5 \times$ first-strand buffer, $5 \mu \mathrm{l}$ $0.1 \mathrm{M}$ DTT, and $1 \mu \mathrm{l}$ RNase OUT, was added. The mixture was then mixed and spun, and placed at room temperature for $2 \mathrm{~min}$. Then, $1 \mu \mathrm{l}$ Superscript II RT was added and gently mixed. The mixture was then spun down and let stand at room temperature for $10 \mathrm{~min}$, incubated at $42{ }^{\circ} \mathrm{C}$ for $50 \mathrm{~min}$, and heat inactivated at $70{ }^{\circ} \mathrm{C}$ for $15 \mathrm{~min}$. Then, $1 \mu \mathrm{l}$ RNase $\mathrm{H}$ was added to the solution, which was gently mixed, spun down, and blocked at $37^{\circ} \mathrm{C}$ for $30 \mathrm{~min}$.

SYBR Green qRT-PCR was performed on the LightCycler $^{\circ} 480$ II System (Roche, Pleasanton, CA) using $5 \mu \mathrm{l}$ $2 \times$ SYBR Green master mix (Roche), $2 \mu$ l RNAse free water, $1 \mu \mathrm{l}$ of forward, $1 \mu \mathrm{l}$ of reverse primer $(10 \mu \mathrm{M})$, and $1 \mu \mathrm{l}$ cDNA per reaction. Primer efficiency was assessed by a dilution series, and a dissociation curve was used to assess primer specificity. qRT-PCR data analysis was performed using genEx software (MultiD).

\section{Statistical analyses}

Percent survival was reported as mean \pm SEM per trial. Every test was repeated at least three times under the same conditions. Survival rate was analyzed by the nonparametric Mann-Whitney test. Correlation analysis between daf-2(rf) allele freeze-thaw survival and lifespan/ other stress traits were conducted by the nonparametric Spearman correlation test.

\section{Availability of supporting data}

Primer sequences,data are accessible through this link: https://mynotebook.labarchives.com/share/fangny/MjMu NHwxMzY4MDYvMTgvVHJIZU5vZGUvMzYxNTQ3Nzk yNXw1OS40.

\section{Additional files}

\begin{abstract}
Additional file 1: Figure S1. daf-2(e1370) enhanced freezing survival under programmed freezing conditions. Reduction-of-function mutant daf-2(e1370) (squares) exhibited significantly increased survival compared with wild-type (N2) animals under the programmed cooling conditions $\left(-1{ }^{\circ} \mathrm{C} / \mathrm{min}\right)$ at different stages. (JPG $259 \mathrm{~kb}$ )
\end{abstract}

Additional file 2: Figure S2. Survival rates of wild-type (N2) and daf-2(e1370rf) under different thawing processes. With different thawing treatments $\left(30^{\circ} \mathrm{C}\right.$ water bath for $1 \mathrm{~min}$ or ice for $30 \mathrm{~min}$ ), wild-type (N2) and daf-2(e1370rf) animals had unchanged survival rates after freeze-thaw stress. (JPG 94 kb)

Additional file 3: Figure S3. Correlated analysis of daf-2(rf) freeze-thaw survival and lifespan, larval arrest, and other stress-resistance traits. Freeze-thaw stress survival phenotypes were not correlated with lifespan, larval arrest, or hypoxia resistance, but was moderately correlated with heat shock survival (JPG $41 \mathrm{~kb}$ )

Additional file 4: Figure S4. Screening for daf-16 target genes required for freezing survival by QF-PCR. daf-16 target genes C01B7.1(sup-37), C36A4.9 (acs-19) , C29A12.3(lig-1) and C46A10.7 (srh-99) have higher expression in daf-2(e1370) compared with wild-type (N2) animals. (JPG 92 kb)

Additional file 5: Table S1. daf-2(rf) allelic variation influence on freeze-thaw stress survival. (JPG 169 kb)

\section{Competing interests}

Authors have no financial and nonfinancial competing interests.

\section{Authors' contributions}

$\mathrm{Hu}$ JP assayed the freezing survival and drafted the manuscript. Xu XY performed the target gene analysis and feeding RNAi . Huang LY measured the daf-16:-GFP and muscle GFP analysis. Wang LS and Fang NY contributed to design the experiment, Wang LS analyzed the data and Fang NY revised the manuscript. All authors read and approved the final manuscript.

\section{Acknowledgements}

This work was supported by grants from the National Natural Science Foundation of China (NSFC81370360,81472758,31170783,U1302225) and Science and Technology Commission of Shanghai (11ZR1421500). All strains were provided by the Caenorhabditis Genetics Center.

Received: 1 August 2015 Accepted: 20 November 2015 Published online: 03 December 2015

\section{References}

1. Ramløv H. Aspects of natural cold tolerance in ectothermic animals. Hum Reprod. 2000;15:26-46.

2. Wharton D. A in Molecular and Physiological Basis of Nematode Survival (eds Perry, R.N. et al.) Ch. 8, 222-223 (Wallingford, 2011).

3. Morris JZ, Tissenbaum HA, Ruvkun G. A phosphatidylinositol-3-OH kinase family member regulating longevity and diapause in Caenorhabditis elegans. Nature. 1996;382:536-9.

4. Paradis S, Ruvkun G. Caenorhabditis elegansAkt/PKB transduces insulin receptor-like signals from age-1 PI3 kinase to the daf-16 transcription factor. Genes Dev. 1998;12:2488-98.

5. Paradis S, Ailion M, Toker A, et al. A PDK1 homolog is necessary and sufficient to transduce age-1 PI3 kinase signals that regulate diapause in Caenorhabditis elegans. Genes Dev. 1999;13:1438-52.

6. Kenyon C, Chang J, Gensch E, et al. C. Elegans mutant that lives twice as long as wild type. Nature. 1993;366:461-4.

7. Ogg S, Ruvkun G. The C. elegans PTEN homolog, DAF-18, acts in the insulin receptor-like metabolic signaling pathway. Mol Cell. 1998;2:887-93. 
8. Gil EB, Link EM, Liu LX, et al. Regulation of the insulin-like developmental pathway of Caenorhabditis elegansby a homolog of the PTEN tumor suppressor gene. Proc Natl Acad Sci U S A. 1999;96:2925-30.

9. Patrick J. Hu, Dauer-wormbook. Available at: http://www.wormbook.org/ chapters/www_dauer/dauer.html

10. Adam Antebi, Nuclear hormone receptors in C. Elegans-wormbook. Available at: http://www.wormbook.org/chapters/www_nuclearhormonerecep/ nuclearhormonerecep.html

11. Ogg S, et al. The Fork head transcription factor daf-16 transduces insulin-like metabolic and longevity signals in C. elegans. Nature. 1997;389:994-9.

12. Savory FR, Sait SM, Hope I. A. daf-16 and Delta9 desaturase genes promote cold tolerance in long-lived Caenorhabditis elegans age-1 mutants. Plos One. 2011;6:e24550.

13. Ohta A, Ujisawa T, Sonoda S, et al. Light and pheromone-sensing neurons regulates cold habituation through insulin signalling in Caenorhabditis elegans. Nat Commun. 2014;22:4412.

14. Hermes-Lima M, Storey KB. Antioxidant defenses in the tolerance of freezing and anoxia by garter snakes. Am J Physiol. 1993;265:R646-52.

15. Mazur P. Cryobiology: the freezing of biological systems. Science. 1970;168:939-49.

16. Toner M, Cravalho EG, Karel M. Cellular response of mouse oocytes to freezing stress: prediction of intracellula ice formation. J Biomech Eng. 1993;115:169-74

17. Finkel T, Holbrook NJ. Oxidants, oxidative stress and the biology of ageing. Nature. 2000:408(6809):239-47.

18. Gems D, Sutton AJ, Sundermeyer ML, et al. Two pleiotropic classes of daf-2mutation affect larval arrest, adult behavior, reproduction and longevity in Caenorhabditis elegans. Genetics. 1998;150(1):129-55.

19. Kimura KD, Tissenbaum HA, Liu Y, et al. daf-2, an insulin receptor-like gene that regulates longevity and diapause in Caenorhabditis elegans. Science. 1997;277(5328):942-6.

20. Fielenbach N, Antebi A. C. elegans dauer formation and the molecular basis of plasticity. Genes Dev. 2008;22:2149-65.

21. Xiao $R$, Zhang $B$, Dong $Y$, et al. A genetic program promotes $C$. elegans longevity at cold temperatures via a thermosensitive TRP channel. Cell. 2013;152(4):806-17.

22. Sakakibara I, Fujino T, Ishii M, et al. Fasting-induced hypothermia and reduced energy production in mice lacking acetyl-CoA synthetase 2. Cell Metab. 2009;9(2):191-202.

23. Riveron J, Boto T, Alcorta E.Transcriptional basis of the acclimation to high environmental temperature at the olfactory receptor organs of Drosophila melanogaster. BMC Genomics.4. doi:10.1186/1471-2164-14-259(2013).

24. Luis H. Toledo-Pereyra. in Organ Preservation for Transplantation 3rd edn, (eds Luis H. Toledo-Pereyra. et al.), Ch.4 45-57, (Landes Bioscience.2010).

25. Lakowski B, Hekimi S. The genetics of caloric restriction in Caenorhabditis elegans. Proc Natl Acad Sci U S A. 1998;95:13091-6.

26. Branicky R, Benard C, Hekimi S. clk-1, mitochondria, and physiological rates. Bioessays. 2000;22:48-56.

27. Feng J, Bussière F, Hekimi S. Mitochondrial electron transport is a key determinant of life span in Caenorhabditis elegans. Dev Cell. 2001;1(5):633-44.

28. Lee SJ, Kenyon C. Regulation of the longevity response to temperature by thermosensory neurons in Caenorhabditis elegans. Curr Biol. 2009;19(9):715-22.

29. Oh SW, Mukhopadhyay A, Dixit BL, et al. Identification of direct daf-16 targets controlling longevity, metabolism and diapause by chromatin immunoprecipitation. Nat Genet. 2005;38:251-7.

30. Lamitina ST, Strange K. Transcriptional targets of daf-16 insulin signaling pathway protect $C$. elegans from extreme hypertonic stress. Am J Physiol Cell Physiol. 2005;288:467-74.

31. McElwee J, Schuster E, Blanc E, Thornton J, Gems D. Diapause-associated metabolic traits reiterated in long-lived daf-2mutants in the nematode Caenorhabditis elegans. Mech Ageing Dev. 2006;127:458-72.

32. Wang J, Kim SK. Global analysis of dauer gene expression in Caenorhabditis elegans. Development. 2003;130:1621-34.

33. Jagdale GB, Grewal PS, Salminen SO. Both heat-shock and cold-shock influence trehalose metabolism in an entomopathogenic nematode. J Parasitol. 2005;91:988-94.

34. Honda Y, Tanaka M, Honda S. Trehalose extends longevity in the nematode Caenorhabditis elegans. Aging Cell. 2010;9:558-69.

35. Murphy CT, McCarroll SA, Bargmann Cl, Fraser A, Kamath RS, et al. Genes that act downstream of daf-16 to influence the lifespan of Caenorhabditis elegans. Nature. 2003;424:277-83.
36. McElwee JJ, Schuster E, Blanc E, Thomas JH, Gems D. Shared transcriptional signature in Caenorhabditis elegans dauer larvae and long-lived daf-2mutants implicates detoxification system in longevity assurance. J Biol Chem. 2004;279:44533-43.

37. Larsen PL. Aging and resistance to oxidative damage in Caenorhabditis elegans. Proc Natl Acad Sci U S A. 1993;90:8905-9.

38. Fontana $L$, Partridge $L$, Longo VD. Extending healthy life span-from yeast to humans. Science. 2010;328:321-6.

39. Kenyon CJ. The genetics of ageing. Nature. 2010;464:504-12.

40. Fire A, Xu S, Montgomery MK, et al. Potent and specific genetic interference by double-stranded RNA in Caenorhabditis elegans. Nature. 1998;391(6669):806-11.

41. Henderson ST, Johnson TE. daf-16 integrates developmental and environmental inputs to mediate aging in the nematode Caenorhabditis elegans. Curr Biol. 2001;11:1975-80.

\section{Submit your next manuscript to BioMed Central and we will help you at every step:}

- We accept pre-submission inquiries

- Our selector tool helps you to find the most relevant journal

- We provide round the clock customer support

- Convenient online submission

- Thorough peer review

- Inclusion in PubMed and all major indexing services

- Maximum visibility for your research

Submit your manuscript at www.biomedcentral.com/submit 